BMJ Open

Diabetes

Research

\& Care

\title{
Trigger finger is associated with risk of incident cardiovascular disease in individuals with type 2 diabetes: a retrospective cohort study
}

\author{
Yusuke Mineoka, ${ }^{1}$ Michiyo Ishii, ${ }^{1}$ Yoshitaka Hashimoto (D) , ${ }^{2}$ Hiroki Yuge, ${ }^{1}$ \\ Machiko Toyoda, ${ }^{1}$ Naoto Nakamura, ${ }^{3}$ Yasukazu Katsumi, ${ }^{4}$ Michiaki Fukui ${ }^{2}$
}

To cite: Mineoka Y, Ishii M, Hashimoto $\mathrm{Y}$, et al. Trigger finger is associated with risk of incident cardiovascular disease in individuals with type 2 diabetes: a retrospective cohort study. BMJ Open Diab Res Care 2021;9:e002070. doi:10.1136/ bmjdrc-2020-002070

Received 11 December 2020 Revised 9 March 2021 Accepted 14 March 2021

\section{Check for updates}

\section{(C) Author(s) (or their} employer(s)) 2021. Re-use permitted under CC BY-NC. No commercial re-use. See rights and permissions. Published by BMJ.

\section{${ }^{1}$ Department of Internal} Medicine, Otsu City Hospital, Otsu, Shiga, Japan

2Department of Endocrinology and Metabolism, Kyoto Prefectural University of Medicine, Graduate School of Medical Science, Kyoto, Japan ${ }^{3}$ Department of Internal Medicine, Saiseikai Kyoto Hospital, Nagaokakyo, Japan ${ }^{4}$ Department of Orthopedics, Takeda Hospital Group, Kyoto, Japan

Correspondence to Dr Michiaki Fukui; michiaki@koto.kpu-m.ac.jp

\section{ABSTRACT}

Introduction Trigger finger is one of the complications affecting the upper extremity in patients with diabetes. Diabetes is also a well-known risk factor that predisposes individuals to cardiovascular diseases (CVDs). This retrospective cohort study aimed to establish the association between trigger finger and the patients with incident CVD with type 2 diabetes.

Materials and methods Trigger finger was diagnosed by palpating a thickened tendon during flexion or on the manifestation of a locking phenomenon during extension or flexion of either finger. The relationship between trigger finger and other clinical parameters or complications of diabetes was examined by a comparative analysis. Cox regression analysis was used to evaluate the association between trigger finger and incidence of CVD. We calculated the propensity scores using sex, body mass index, age, smoking status, duration of diabetes, estimated glomerular filtration rate, hypertension, dyslipidemia, and hemoglobin A1c as the number of patients with incident CVD during the follow-up period was low.

Results Among the 399 patients with type 2 diabetes, 54 patients had trigger finger. Patients with trigger finger were significantly older in age and had been suffering from diabetes for a longer duration. They also displayed worse renal function and glycemic control, along with a higher incidence of hypertension, neuropathy and nephropathy. During the average $5.66 \pm 1.12$ years of follow-up, a total of 18 incidents occurred. According to the Cox regression analysis, trigger finger was shown to be associated with enhanced risk of the incidence of CVD after adjustment for the covariates (adjusted $\mathrm{HR}=3.33(95 \% \mathrm{Cl} 1.25$ to 8.66), $\mathrm{p}=0.017$ ).

Conclusions Trigger finger is associated with the risk of incident CVD in patients with type 2 diabetes. Thus, clinicians must consider these factors at the time of diagnosis of such patients.

\section{INTRODUCTION}

Cardiovascular disease (CVD) is the most prevalent cause of morbidity and mortality in patients with diabetes. ${ }^{1}$ Type 2 diabetes is a known risk factor for atherosclerosis, leading to non-embolic ischemic stroke ${ }^{2}$ and peripheral arterial disease. ${ }^{3}$ To prevent CVD,

\section{Significance of this study}

What is already known about this subject?

- Diabetic microvascular complications are associated with the risk of cardiovascular disease (CVD).

- There is an association between diabetic hand and the presence of macroangiopathy in cross-sectional manners.

\section{What are the new findings?}

- Patients with trigger finger were older, had a longer disease duration, and had a higher prevalence of hypertension, neuropathy and nephropathy.

- Trigger finger is associated with the risk of incident CVD in patients with type 2 diabetes.

How might these results change the focus of research or clinical practice?

- Clinicians need to be aware of the prevalence of trigger finger as it is associated with a higher incidence of CVD, since diagnosis of trigger finger is relatively easy and non-invasive.

cardiovascular risk factors including hypertension, dyslipidemia, chronic kidney disease, and the presence of diabetic complications such as microangiopathy should be assessed annually in all patients with diabetes. ${ }^{4}$

Trigger finger, a common distressing condition of the hand, is associated with organ overuse and is considered a complication of the upper extremity in affected individuals. It is also referred to as a form of 'diabetic hand', like limited joint mobility (LJM) and Dupuytren's contracture. ${ }^{5-7}$ In patients with diabetes, the prevalence of trigger finger is more common (20\%) than the general population $(1 \%-2 \%){ }^{8}{ }^{9}$ This complication was often ignored in the clinical settings, ${ }^{10}$ although several reports have revealed that diabetic hand is associated with microvascular complications. ${ }^{5711}$ 
Earlier, only a minuscule number of reports have discussed the association between diabetic hand and the presence of macroangiopathy in cross-sectional manners: patients with diabetes with LJM were more likely to had thickened intima-media thickness and had a history of coronary heart and cerebrovascular disease.${ }^{12-14}$ However, it remains to be elucidated whether there exists a relationship between trigger finger and the incident CVD in patients with type 2 diabetes mellitus. To this effect, the present retrospective cohort study was undertaken to comprehend this relationship.

\section{MATERIALS AND METHODS}

\section{Study design and patients}

Patients with type 2 diabetes who were over 20 years of age were included in this retrospective cohort study. For this study, outpatients at Otsu City Hospital between April 2013 and December 2017 were recruited. The exclusion criteria are as follows: patients with a history of articular rheumatism or surgery for the disease of the cervical spine, patients with severe renal (estimated glomerular filtration rate (eGFR) $<30 \mathrm{~mL} / \mathrm{min} / 1.73 \mathrm{~m}^{2}$ ) or liver failure (exhibiting liver cirrhosis, aspartate aminotransferase and/or alanine aminotransferase $\geq 2.5$ times the normal range) and patients with a history of CVD, including myocardial infarction, coronary revascularisation and/or stroke).

Data on demographics, medical history, and medication use of all patients were gathered. According to the selfadministered questionnaire, patients were classified into either current smokers or non-smokers. The body mass index (BMI) was calculated as weight ( $\mathrm{kg}$ ) divided by height squared $\left(\mathrm{m}^{2}\right)$.

The retinopathy was assessed by ophthalmologists who were not informed on the status of diabetes in the patients. ${ }^{15}$ Neuropathy was assessed on the basis of the diagnostic criteria for diabetic neuropathy as proposed by the Diagnostic Neuropathy Study Group. ${ }^{16}$ Nephropathy was graded as normoalbuminuria when urine albumin excretion (UAE) was $<30 \mathrm{mg} / \mathrm{g}$ creatinine $(\mathrm{Cr})$; as microalbuminuria when UAE was within the range of $30-300 \mathrm{mg} / \mathrm{g} \mathrm{Cr}$, and as macroalbuminuria, when UAE was $\geq 300 \mathrm{mg} / \mathrm{g}$ Cr. The incidence of nephropathy was assumed in the case of microalbuminuria or macroalbuminuria.

\section{Data collection and measurements}

Blood examinations were conducted using a Bio Majesty JCA-BM 6050 (JEOL, Tokyo, Japan). All blood samples were drawn in the morning after the subjects had completed an overnight fast for the measurement of glycated hemoglobin (HbAlc), total cholesterol, triglycerides, and Cr.

Hypertension was defined as systolic blood pressure $\geq 140 \mathrm{~mm} \mathrm{Hg}$, diastolic blood pressure $\geq 90 \mathrm{~mm} \mathrm{Hg}$, and/ or having received treatment for hypertension. Dyslipidemia was considered when total cholesterol concentration $>5.69 \mathrm{mmol} / \mathrm{L}$, triglycerides concentration $>1.70 \mathrm{mmol} / \mathrm{L}$, and/or when patients received treatment for dyslipidemia.
The eGFR was calculated as follows ${ }^{17}$ : male: eGFR $\left(\mathrm{mL} / \mathrm{min} / 1.73 \mathrm{~m}^{2}\right)=194 \times$ age $^{-0.287} \times(\text { serum } \mathrm{Cr})^{-1.094}$; and female: eGFR $=194 \times$ age $^{-0.287} \times(\text { serum Cr })^{-1.094} \times 0.739$.

\section{Diagnosis of trigger finger}

Trigger finger was diagnosed by palpating a thickened flexion tendon or upon the manifestation of a locking phenomenon during extension or flexion of either finger. ${ }^{18}$

\section{Definition of CVD}

Incident CVD was defined as any first-time inpatient admission with any of the CVD such as myocardial infarction, unstable angina, heart failure and stroke assessed with medical records reviewed by physicians. Diagnosis of CVD was confirmed by specialists using the International Classification of Disease-10 code during the study period.

\section{Statistical analysis}

Statistical analyses were performed using JMP V.9.0 and V.11 (SAS Institute, Cary, North Carolina, USA). P value of $<0.05$ was considered statistically significant.

Categorical variables were presented as number $(\%)$. Continuous variables were presented as the mean value $\pm 1 \mathrm{SD}$. The significance of differences between the group was assessed using unpaired Student's t-test, a Pearson $\chi^{2}$ test or analyses of variance.

Kaplan-Meier analysis was performed to obtain a graphical representation of time to incident CVD, and a log-rank test was performed to assess the difference between patients with trigger finger and those without it.

The HR for the risk of incident CVD was calculated using a Cox regression model. Because of the small number of patients' incident CVD, we calculated a propensity score. To calculate propensity score, multiple multivariate logistic regression analysis was performed to combined covariates, age, sex, duration of diabetes, Cr level, BMI, current smoking status, hypertension, status of dyslipidemia, and HbAlc level as predictor variables, with trigger finger taken as the dependent variable (the C-statistic was 0.73 ) (model 1). We also performed multiple multivariate logistic regression analysis to combined covariates, age, sex, duration of diabetes, eGFR, ${ }^{19}$ BMI, current smoking status, hypertension, status of dyslipidemia, and $\mathrm{HbAlc}$ level as predictor variables, with trigger finger taken as the dependent variable (the C-statistic was 0.73) (model 2). Then, the HR for the risk of incident CVD was calculated using a Cox regression model with trigger finger and propensity score.

\section{RESULTS}

In this retrospective cohort study, 498 patients with type 2 diabetes were included while 47 patients were excluded as they reported a history of cardiovascular events. Another 40 subjects were excluded due to reported instances of surgery for spinal disease or articular rheumatism. A total of nine individuals were excluded due to renal insufficiency and three were excluded due to liver failure.

The clinical characteristics of the remaining 399 patients with type 2 diabetes (226 men and 173 women) have been 
Table 1 Clinical characteristics of patients with or without trigger finger

\begin{tabular}{lllr}
\hline & $\begin{array}{l}\text { Trigger } \\
\text { finger- } \\
\mathbf{n = 3 4 5}\end{array}$ & $\begin{array}{l}\text { Trigger } \\
\text { finger+ } \\
\mathbf{n = 5 4}\end{array}$ & P value \\
\hline Age (years) & $62.3 \pm 14.6$ & $69.7 \pm 10.5$ & $<0.001$ \\
\hline Male (\%) & 57.7 & 50.0 & 0.094 \\
\hline Duration of diabetes (year) & $12.1 \pm 10.6$ & $16.5 \pm 11.2$ & 0.006 \\
\hline BMI (kg/m ${ }^{2}$ ) & $24.9 \pm 4.7$ & $25.2 \pm 5.7$ & 0.646 \\
\hline Hemoglobin A1c (\%) & $7.3 \pm 1.1$ & $7.7 \pm 1.4$ & 0.024 \\
\hline Creatinine ( $\mu$ mol/L) & $70.2 \pm 30.6$ & $76.9 \pm 58.3$ & 0.264 \\
\hline eGFR (mL/min/1.73 $\mathrm{m}^{2}$ ) & $71.3 \pm 23.6$ & $64.5 \pm 21.5$ & 0.025 \\
\hline Hypertension (\%) & 50.3 & 68.5 & 0.041 \\
\hline Dyslipidemia (\%) & 50.1 & 53.1 & 0.555 \\
\hline Smoking (\%) & 5.8 & 11.0 & 0.282 \\
\hline Neuropathy (\%) & 22.0 & 48.1 & $<0.001$ \\
\hline Nephropathy (\%) & 37.9 & 55.5 & 0.043 \\
\hline Retinopathy (\%) & 23.7 & 37.0 & 0.065 \\
\hline
\end{tabular}

Continuous variables are presented as the mean value $\pm 1 \mathrm{SD}$. Categorical variables are presented as a number (percentage).

$\mathrm{BMI}$, body mass index; eGFR, estimated glomerular filtration rate.

indicated in table 1 . Of the total number of patients, 54 individuals $(13.5 \%)$ had trigger finger and these patients were significantly older who have had diabetes for a longer duration of time. These patients also had worse glycemic control, hypertension, neuropathy and nephropathy when compared with subjects who did not have trigger finger (table 1). In patients who excluded with the history of CVD, the prevalence of trigger finger was $19 \%(n=9 / 47)$ and was tended to be higher than study populations, although the difference did not reach statistical significance $(\mathrm{p}=0.283)$.

During the average 5.66 \pm 1.12 years of the follow-up period, 18 patients developed CVD such as stroke $(n=6)$, unstable angina $(n=5)$, heart failure $(n=5)$ and myocardial infarction $(n=2)$. No patients died from noncardiovascular events, including cancer.
Log-rank test revealed that the cumulative rates of incident CVD were $13.0 \%$ in patients with trigger finger and $3.2 \%$ in patients without trigger finger $(\mathrm{p}=0.006)$ (figure 1$)$. According to the univariate Cox regression analysis, age, duration of diabetes, $\mathrm{Cr}$ and trigger finger are associated with patients who developed CVD (table 2). A multivariate Cox regression analysis with calculated propensity scores of combined covariates revealed that trigger finger was associated with an increased risk of incident CVD (model 1, HR=3.15 (95\% CI 1.06 to 8.87), $\mathrm{p}=0.038$ and model 2 , $\mathrm{HR}=3.33$ (95\% CI 1.25 to 8.66$), \mathrm{p}=0.017$ ).

\section{DISCUSSION}

This retrospective cohort study investigated the relationship between trigger finger and the risk of developing CVD in patients with type 2 diabetes, and indicated that trigger finger was associated with the increased risk of incident CVD.

In patients with type 2 diabetes, CVD is a critical risk that could lead to death and disability. Thus, management of both diabetes and CVD with good glycemic control is an important issue. In patients with type 1 diabetes, the importance of glycemic control has been studied well that aids in the prevention of microvascular complications and CVD. ${ }^{2021}$ In patients with type 2 diabetes, tight glycemic control does not always give a good result in the prevention of CVD. ${ }^{22}$ Thus, in order to prevent CVD in patients with type 2 diabetes, recognizing and controlling diabetic complications and other several risk factors are important such as hypertension, dyslipidemia, smoking status and obesity in addition to the glycemic status of the affected individuals. ${ }^{23-26}$

In this study, patients with trigger finger displayed a higher propensity (four times) of incident CVD when compared with patients without trigger finger. Older age, longer duration of diabetes, worse renal function, higher proportion of hypertension and microvascular complications were observed in patients with trigger finger. These might be common background factors about trigger finger and

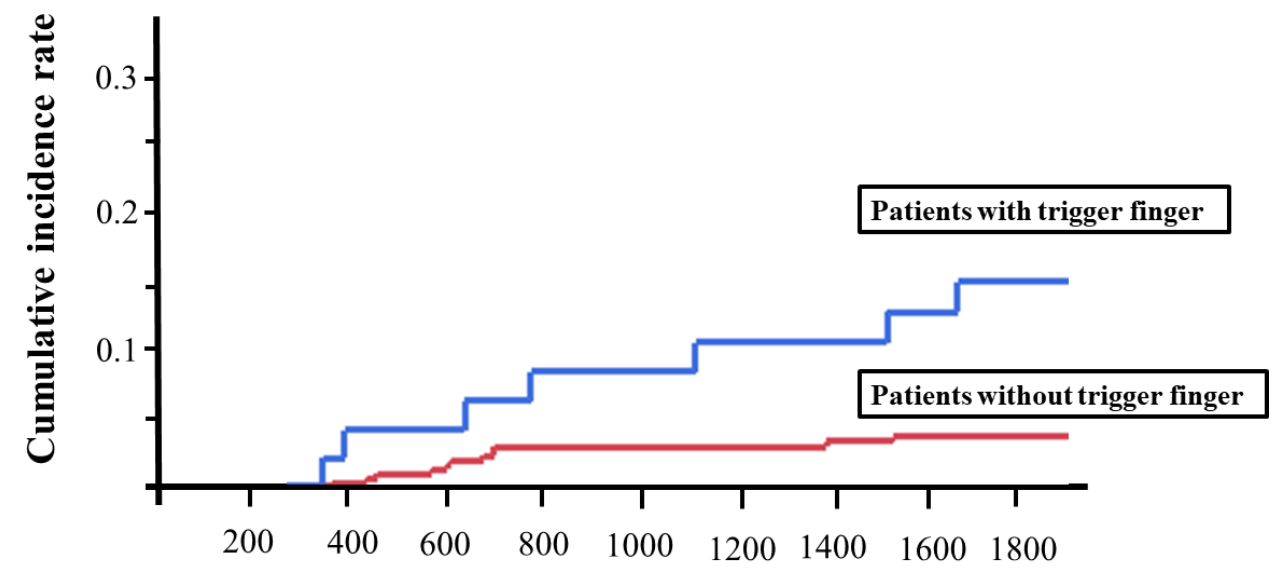

Days for hospitalization with cardiovascular disease

Figure 1 Probability of cumulative incident cardiovascular disease. Vertical axis represents cumulative incidence rate; horizontal axis represents days of incident cardiovascular disease. Blue line represents patients with trigger finger; red line represents patients without trigger finger. 
Table 2 Univariate HR $(95 \% \mathrm{Cl})$ for hospitalization with CVD

\begin{tabular}{llr}
\hline & \multicolumn{2}{l}{ Univariate analysis } \\
\cline { 2 - 3 } & HR $(95 \%$ Cl) & P value \\
\hline Age (years) & $1.06(1.02$ to 1.11$)$ & 0.004 \\
\hline Duration of diabetes (year) & $1.05(1.01$ to 1.09$)$ & 0.012 \\
\hline BMI (kg/m $\left.{ }^{2}\right)$ & $0.91(0.83$ to 1.01$)$ & 0.085 \\
\hline Hemoglobin A1c $(\%)$ & $1.10(0.73$ to 1.56$)$ & 0.625 \\
\hline Male & $1.33(0.51$ to 3.48$)$ & 0.554 \\
\hline Dyslipidemia $(\%)$ & $1.36(0.52$ to 3.65$)$ & 0.518 \\
\hline Smoking & $1.40(0.27$ to 3.67$)$ & 0.736 \\
\hline Creatinine $(\mathrm{mg} / \mathrm{dL})$ & $2.71(1.38$ to 6.43$)$ & 0.005 \\
\hline eGFR (mL/min/1.73 $\left.\mathrm{m}^{2}\right)$ & $0.96(0.94$ to 0.98$)$ & $<0.001$ \\
\hline Trigger finger & $4.52(1.60$ to 12.1$)$ & 0.006 \\
\hline
\end{tabular}

Data are expressed as mean $\pm \mathrm{SD}$.

BMI, body mass index; CVD, cardiovascular disease; eGFR, estimated glomerular filtration rate.

macroangiopathy or CVD. ${ }^{727}$ Moreover, recent studies have investigated the mechanisms common to macroangiopathy and trigger finger such as advanced glycation end products (AGEs) and calcification.

AGEs are one of the mechanisms that contribute to the occurrence of atherosclerosis in patients with diabetes. ${ }^{28}$ AGEs are formed and accumulated in circulating blood and several tissues in chronic hyperglycemic status. ${ }^{29}{ }^{30}$ AGEs are recognized by a cell surface receptor AGE (RAGE) which might induce oxidative stress and inflammatory mediator, thereby leading to the onset and progression of diabetic microangiopathy and macroangiopathy. ${ }^{31}{ }^{32}$ Similar changes mediated by AGE-RAGE interaction are thought to be the mechanism behind the incidence of trigger finger. AGEs form covalent cross-links within collagen fibers that contribute to an alteration in the structure and function of tendons. ${ }^{2830}$

Calcification of blood vessels in patients with diabetes is promoted by AGEs that is formed in the arterial wall collagen. ${ }^{32}$ The molecular structure of the AGEs-modified collagen is changed to facilitate calcium deposition. ${ }^{32}$ Calcifications of tendons in patients with diabetes have also been reported. ${ }^{33}$ Other mechanisms such as overproduction of growth factors and cytokines such as transforming growth factor-beta are also believed to increase collagen deposition in the skin and musculoskeletal connective tissues of the hand. ${ }^{34}$ These mechanisms mentioned above might be associated with both trigger finger and macrovascular complications of diabetes. ${ }^{35-38}$ In this study, patients with trigger finger were more likely to have hypertension. This result was similar to other studies. ${ }^{38}{ }^{39}$ Microvascular disease has been reported to affect tendon damage ${ }^{38}$ therefore, there is a possibility that tight medication for well-known risk factors for microvascular disease might result in preventing trigger finger.

This study showed the association between trigger finger and the risk of CVD in patients with type 2 diabetes. The diagnosis of trigger finger might be the weakness of this study, since there is a possibility that the severe symptom of the hand is associated with diabetic complications including microangiopathy. In fact, previous study on patients with type 1 diabetes shows that collagen accumulates in connective tissue and the amount of collagen cross-linking correlates with the disease duration, skin changes and limitation of joint movements. ${ }^{40}$ However, trigger finger has been recognized over the years and is relatively easy to assess in clinical situations.

There are several limitations in this study. First, this study was a retrospective study, and therefore we cannot deny the possibility of the influences by unknown confounding factors. Second, it was difficult to exclude some patients with trigger finger because of their tough job or overuse of their hand. Third, Dupuytren's disease and carpal tunnel syndrome are also components of 'diabetic hand' and were informative in clinical situation. Unfortunately, however, we could not investigate them in this study. Fourth, the outcome of this study is based on soft endpoint because of the retrospective design. Finally, the number of participants in this study was relatively small and all were from the same country, Japan. The prevalence rate of trigger finger in this study was different from other studies, ${ }^{8941}$ and was similar between men and women. This might be because of varying study population characteristics such as age, duration of diabetes, comorbidities and ethnicity. Many older patients were included in this study. In older patients, the prevalence of trigger finger was high for both men and women. ${ }^{9}$ Thus, it is uncertain if our finding could be applicable to other populations.

In conclusion, our report indicated that trigger finger is related to the incidence of CVD in patients with type 2 diabetes. Trigger finger is well known, and its diagnosis is relatively easy and non-invasive. Therefore, we should be aware of the prevalence of trigger finger and that it is associated with a higher chance of developing CVD in patients with type 2 diabetes.

Acknowledgements The authors thank all the staff members of the diabetes team in Otsu City Hospital for their assistance. They also would like to thank Editage (www.editage.com) for English language editing.

Contributors YM contributed to the conception and design of the study, research and analysis, and writing the manuscript. Ml contributed to acquisition of data, critical revision of the manuscript, and analysis and interpretation of data. $\mathrm{YH}$ contributed to critical revision of the manuscript as well as analysis and interpretation of data. HY and MT contributed to acquisition of data and revision of the manuscript. NN and YK contributed to the conception of the study and revision of the manuscript. MF contributed to analysis and interpretation of data, and critica revision of the manuscript. All authors approved the final version of the manuscript and agreed to be accountable for all aspects of the work in ensuring that questions related to the accuracy or integrity of any part of the work are appropriately investigated and resolved.

Funding The authors have not declared a specific grant for this research from any funding agency in the public, commercial or not-for-profit sectors.

Competing interests $\mathrm{YH}$ received grants from Asahi Kasei Pharma outside the submitted work. MF received grants from Taisho Pharma Co, Daiichi Sankyo Co, MSD, Sumitomo Dainippon Pharma Co, Novo Nordisk Pharma, Kissei Pharma Co, Mitsubishi Tanabe Pharma Corp, Astellas Pharma, Nippon Boehringer Ingelheim Co, Sanwa Kagagu Kenkyusho Co, Tejin Pharma, Takeda Pharma Co, Sanofi, Kowa Pharma Co, Kyowa Kirin Co, Ono Pharma Co, Eli Lilly Japan, Nippon Chemiphar Co, Johnson \& Johnson Medical Co, Abbott Japan Co, and Terumo Corp; and received honoraria from Mitsubishi Tanabe Pharma Corp, Sanofi, Novo Nordisk Pharma, MSD, AstraZeneca, Kowa Pharma Co, Ono Pharma Co, Sanwa Kagaku Kenkyusho Co, Mochida Pharma Co, Takeda Pharma Co, Astellas Pharma, Teijin Pharma, 
Sumitomo Dainippon Pharma Co, Daiichi Sankyo Co, Nippon Boehringer Ingelheim Co, Taisho Pharma Co, Bayer Yakuhin, Abbott Japan Co, Eli Lilly Japan, Medtronic Japan Co, Arkray, Kissei Pharma Co, Kyowa Kirin Co, and Nipro Corp outside the submitted work.

\section{Patient consent for publication Not required.}

Ethics approval This study was conducted in accordance with the Declaration of Helsinki, and informed consent was obtained from all patients. The Ethics Committee of the Otsu City Hospital approved this study (no. 213).

Provenance and peer review Not commissioned; externally peer reviewed.

Data availability statement Data are available upon reasonable request.

Open access This is an open access article distributed in accordance with the Creative Commons Attribution Non Commercial (CC BY-NC 4.0) license, which permits others to distribute, remix, adapt, build upon this work non-commercially, and license their derivative works on different terms, provided the original work is properly cited, appropriate credit is given, any changes made indicated, and the use is non-commercial. See: http://creativecommons.org/licenses/by-nc/4.0/.

ORCID iD

Yoshitaka Hashimoto http://orcid.org/0000-0002-8794-0550

\section{REFERENCES}

1 Isomaa B, Almgren P, Tuomi T, et al. Cardiovascular morbidity and mortality associated with the metabolic syndrome. Diabetes Care 2001;24:683-9.

2 Iso $\mathrm{H}$, Imano $\mathrm{H}$, Kitamura $\mathrm{A}$, et al. Type 2 diabetes and risk of non-embolic ischaemic stroke in Japanese men and women. Diabetologia 2004;47:2137-44.

3 Criqui MH. Peripheral arterial disease--epidemiological aspects. Vasc Med 2001;6:3-7.

4 American Diabetes Association. 10. cardiovascular disease and risk management: standards of medical care in diabetes-2019. Diabetes Care 2019;42:S103-23.

5 Rosenbloom AL, Silverstein JH, Lezotte DC, et al. Limited joint mobility in childhood diabetes mellitus indicates increased risk for microvascular disease. N Engl J Med 1981;305:191-4.

6 Jennings AM, Milner PC, Ward JD. Hand abnormalities are associated with the complications of diabetes in type 2 diabetes. Diabet Med 1989;6:43-7.

7 Crispin JC, Alcocer-Varela J. Rheumatologic manifestations of diabetes mellitus. Am J Med 2003;114:753-7.

8 Chammas M, Bousquet P, Renard E, et al. Dupuytren's disease, carpal tunnel syndrome, trigger finger, and diabetes mellitus. J Hand Surg Am 1995;20:109-14

9 Shen P-C, Chang P-C, Jou I-M, et al. Hand tendinopathy risk factors in Taiwan: a population-based cohort study. Medicine 2019;98:e13795.

10 Papanas N, Maltezos E. The diabetic hand: a forgotten complication? J Diabetes Complications 2010;24:154-62.

11 Mineoka Y, Ishii M, Tsuji A, et al. Relationship between limited joint mobility of the hand and diabetic foot risk in patients with type 2 diabetes. J Diabetes 2017:9:628-33.

12 Arkkila PE, Kantola IM, Viikari JS. Limited joint mobility in noninsulin-dependent diabetic (NIDDM) patients: correlation to control of diabetes, atherosclerotic vascular disease, and other diabetic complications. J Diabetes Complications 1997;11:208-17.

13 Frost $\mathrm{D}$, Beischer W. Limited joint mobility in type 1 diabetic patients: associations with microangiopathy and subclinical macroangiopathy are different in men and women. Diabetes Care 2001;24:95-9.

14 Mineoka Y, Ishii M, Hashimoto Y, et al. Relationship between limited joint mobility of hand and carotid atherosclerosis in patients with type 2 diabetes. Diabetes Res Clin Pract 2017;132:79-84.

15 Tanaka K, Kawai T, Saisho Y, et al. Relationship between stage of diabetic retinopathy and pulse wave velocity in Japanese patients with type 2 diabetes. J Diabetes Res 2013;2013:1-4.

16 Yasuda H, Sanada M, Kitada K, et al. Rationale and usefulness of newly devised abbreviated diagnostic criteria and staging for diabetic polyneuropathy. Diabetes Res Clin Pract 2007;77 Suppl $1: \mathrm{S} 178-83$
17 Matsuo S, Imai E, Horio M, et al. Revised equations for estimated GFR from serum creatinine in Japan. Am J Kidney Dis 2009;53:982-92.

18 Ryzewicz M, Wolf JM. Trigger digits: principles, management, and complications. J Hand Surg Am 2006;31:135-46.

19 Chronic Kidney Disease Prognosis Consortium, Matsushita K, van der Velde $\mathrm{M}$, et al. Association of estimated glomerular filtration rate and albuminuria with all-cause and cardiovascular mortality in general population cohorts: a collaborative meta-analysis. Lancet 2010;375:2073-81.

20 Diabetes Control and Complications Trial Research Group, Nathan DM, Genuth S, et al. The effect of intensive treatment of diabetes on the development and progression of long-term complications in insulin-dependent diabetes mellitus. N Engl J Med 1993;329:977-86.

21 Nathan DM, Cleary PA, Backlund J-YC, et al. Intensive diabetes treatment and cardiovascular disease in patients with type 1 diabetes. N Engl J Med 2005;353:2643-53.

22 ADVANCE Collaborative Group, Patel A, MacMahon S, et al. Intensive blood glucose control and vascular outcomes in patients with type 2 diabetes. N Engl J Med 2008;358:2560-72.

23 Intensive blood-glucose control with sulphonylureas or insulin compared with conventional treatment and risk of complications in patients with type 2 diabetes (UKPDS 33). UK prospective diabetes study (UKPDS) group. Lancet 1998;352:837-53.

24 Action to Control Cardiovascular Risk in Diabetes Study Group, Gerstein HC, Miller ME, et al. Effects of intensive glucose lowering in type 2 diabetes. N Engl J Med 2008;358:2545-59.

25 Kawasaki R, Tanaka S, Tanaka S, et al. Risk of cardiovascular diseases is increased even with mild diabetic retinopathy: the Japan diabetes complications study. Ophthalmology 2013;120:574-82.

26 Gaede $\mathrm{P}$, Lund-Andersen $\mathrm{H}$, Parving $\mathrm{H}-\mathrm{H}$, et al. Effect of a multifactorial intervention on mortality in type 2 diabetes. $N$ Engl J Med 2008;358:580-91.

27 Kiani J, Goharifar H, Moghimbeigi A, et al. Prevalence and risk factors of five most common upper extremity disorders in diabetics. $J$ Res Health Sci 2014;14:92-5.

28 Goldin A, Beckman JA, Schmidt AM, et al. Advanced glycation end products: sparking the development of diabetic vascular injury. Circulation 2006;114:597-605.

29 Yamagishi S-ichi, Nakamura K, Imaizumi T. Advanced glycation end products (AGEs) and diabetic vascular complications. Curr Diabetes Rev 2005;1:93-106.

30 Vazzana N, Santilli F, Cuccurullo C, et al. Soluble forms of RAGE in internal medicine. Intern Emerg Med 2009;4:389-401.

31 Yamagishi S-ichi, Nakamura K, Matsui T, et al. Receptor for advanced glycation end products (RAGE): a novel therapeutic target for diabetic vascular complication. Curr Pharm Des 2008;14:487-95.

32 Sakata N, Takeuchi K, Noda K, et al. Calcification of the medial layer of the internal thoracic artery in diabetic patients: relevance of glycoxidation. J Vasc Res 2003;40:567-74.

33 Oliva F, Via AG, Maffulli N. Physiopathology of intratendinous calcific deposition. BMC Med 2012;10:95.

34 Miyauchi K, Takiyama Y, Honjyo J, et al. Upregulated IL-18 expression in type 2 diabetic subjects with nephropathy: TGF-beta1 enhanced IL-18 expression in human renal proximal tubular epithelia cells. Diabetes Res Clin Pract 2009;83:190-9.

35 Smith LL, Burnet SP, McNeil JD. Musculoskeletal manifestations of diabetes mellitus. Br J Sports Med 2003;37:30-5.

36 Ravindran Rajendran S, Bhansali A, Walia R, et al. Prevalence and pattern of hand soft-tissue changes in type 2 diabetes mellitus. Diabetes Metab 2011;37:312-7.

37 Arkkila PET, Gautier J-F. Musculoskeletal disorders in diabetes mellitus: an update. Best Pract Res Clin Rheumatol 2003;17:945-70.

38 Abate M, Schiavone C, Salini V, et al. Management of limited joint mobility in diabetic patients. Diabetes Metab Syndr Obes 2013;6:197-207.

39 Mustafa KN, Khader YS, Bsoul AK, et al. Musculoskeletal disorders of the hand in type 2 diabetes mellitus: prevalence and its associated factors. Int J Rheum Dis 2016;19:730-5.

40 Snedeker JG, Gautieri A. The role of collagen crosslinks in ageing and diabetes - the good, the bad, and the ugly. Muscles Ligaments Tendons J 2014;4:303-8.

41 Pandey A, Usman K, Reddy $\mathrm{H}$, et al. Prevalence of hand disorders in type 2 diabetes mellitus and its correlation with microvascular complications. Ann Med Health Sci Res 2013;3:349-54. 